\title{
Self-similar evolution of wind-blown bubbles with mass loading by hydrodynamic ablation
}

\author{
J. M. Pittard, T. W. Hartquist, and J. E. Dyson
}

Department of Physics \& Astronomy, The University of Leeds, Woodhouse Lane, Leeds, LS2 9JT, UK

Received 6 March 2001 / Accepted 2 May 2001

\begin{abstract}
We present similarity solutions for adiabatic bubbles that are blown by winds having time independent mechanical luminosities and that are each mass-loaded by the hydrodynamic ablation of distributed clumps. The mass loading is "switched-on" at a specified radius (with free-expansion of the wind interior to this point) and injects mass at a rate per unit volume proportional to $M^{\delta} r^{\lambda}$ where $\delta=4 / 3$ (1) if the flow is subsonic (supersonic) with respect to the clumps. In the limit of negligible mass loading a similarity solution found by Dyson (1973) for expansion into a smooth ambient medium is recovered. The presence of mass loading heats the flow, which leads to a reduction in the Mach number of the supersonic freely-expanding flow, and weaker jump conditions across the inner shock. In solutions with large mass loading, it is possible for the wind to connect directly to the contact discontinuity without first passing through an inner shock, in agreement with previous hydrodynamic simulations. In such circumstances, the flow may or may not remain continuously supersonic with respect to the clumps. For a solution that gives the mass of swept-up ambient gas to be less than the sum of the masses of the wind and ablated material, $\lambda \lesssim-2$, meaning that the exponent of the density profile of the interclump medium must be at most slightly positive, with negative values preferred. Maximum possible values for the ratio of ablated mass to wind mass occur when mass loading starts very close to the bubble center and when the flow is supersonic with respect to the clumps over the entire bubble radius. Whilst mass loading always reduces the temperature of the shocked wind, it also tends to reduce the emissivity in the interior of the bubble relative to its limb, whilst simultaneously increasing the central temperature relative to the limb temperature. The maximum temperature in the bubble often occurs near the onset of mass loading, and in some cases can be many times greater than the post-inner-shock temperature. Our solutions are potentially relevant to a wide range of astrophysical objects, including stellar wind-blown bubbles, galactic winds, starburst galaxy superwinds, and the impact of an AGN wind on its surrounding environment. This work complements the earlier work of Pittard et al. (2001) in which it was assumed that clumps were evaporated through conductive energy transport.
\end{abstract}

Key words. hydrodynamics - shock waves - stars: mass-loss - ISM: bubbles - galaxies: active

\section{Introduction}

Numerous observations of wind-blown bubbles (WBBs) have led to the conclusion that their structures and evolution are significantly affected by mass entrainment from embedded clumps. As a case in point, bubbles blown by the winds of highly evolved stars can be mass-loaded from clumps of stellar material ejected during prior stages of massloss (Smith et al. 1984; Hartquist et al. 1986; Meaburn et al. 1991; Dyson \& Hartquist 1992; Hartquist \& Dyson 1993). The properties of winds mass-loaded by material from clumps (or stellar sources) has also received theoretical attention in the context of ultracompact H II regions (Dyson et al. 1995), globular cluster winds (Durisen \& Burns 1981), galactic winds and starburst galaxy superwinds (Strickland \& Stevens 2000), and accretion flow

Send offprint requests to: J. M. Pittard, e-mail: jmp@ast.leeds.ac.uk structures at the centers of active galactic nuclei (David \& Durisen 1989; Toniazzo et al. 2001).

Investigations into the self-similar nature of supernova remnant (McKee \& Ostriker 1977; Chièze \& Lazareff 1981; Dyson \& Hartquist 1987) and stellar wind-blown bubble (Pittard et al. 2001) evolution in tenuous media with embedded clumps have been performed. Detailed one-dimensional, time-dependent hydrodynamic models of specific WBBs associated with evolved stars have also been constructed (Arthur et al.1993, 1994, 1996). In at least some isothermal mass-loaded winds, Arthur et al. (1994) and Williams et al. $(1995,1999)$ showed that a global shock does not occur in the region where the Mach number is large. Instead, a global shock occurs only after the wind has travelled far enough for mass loading to decelerate it sufficiently that its Mach number is less than a few. In some of these cases, a global shock within the mass loading region does not exist at all, and the wind 
continues until it encounters a termination shock caused by the interaction of the wind with an external medium.

Observations supporting mass loading in WBBs include the detection of blue-shifted absorption features of species with a range of ionization potentials in the spectrum of the central star of the Wolf-Rayet nebula RCW 58. Smith et al. (1984) argued that the observed velocity spread for the detected features is much larger than would be expected for $T \lesssim 10^{5} \mathrm{~K}$ gas consisting only of stellar wind material decelerated through a terminal shock, and suggested that the velocity spread originates due to mass loading of the shocked wind through the entrainment of material from clumps. Crucially, one can obtain an estimate for the ratio of wind mass to entrained clump mass: the observed velocity spread implies a value of 40 or 50 to one. A detailed time-dependent, hydrodynamic model including nonequilibrium ionization structure is consistent with these conclusions (Arthur et al. 1996). Spectroscopic data also provide evidence for transonic flows in the halo of core-halo planetary nebula (Meaburn et al. 1991), again consistent with high mass loading rates. Finally, it is also suspected that the gradual deceleration of a wind by mass loading and the associated weakening of an inner shock may in fact contribute to the radio quietness of some WBBs, although this is a conjecture which currently cannot be proven (see Williams et al. 1995, 1999).

In Pittard et al. (2001, hereafter PDH), similarity solutions were derived for the structures and evolution of mass-loaded WBBs, under the assumption that conductive evaporation from embedded clumps was the dominant mass loading process, and that both evaporation from the cold swept-up shell and radiative losses were negligible. To obtain a similarity solution with these conditions, specific radial power-laws on the clump and interclump density distribution, and temporal power-laws on the wind mass-loss rate and terminal velocity, were required. Approximate similarity solutions for evaporatively mass-loaded WBBs with the assumptions of constant mass-loss rate and wind velocity, and an isobaric shocked wind region have previously been obtained by Weaver et al. (1977; evaporation from the cool swept-up shell) and Hanami \& Sakashita (1987; mass-loading from clumps). A central assumption in both of these papers was that the shocked wind was approximately isobaric. However, PDH demonstrated that this was not necessarily a good assumption (e.g. see their Fig. 4). Indeed, imposing this condition is likely to set a limit to the amount of mass loading that can occur.

A central conclusion of PDH was that there exist maximum possible values for the ratio of evaporated mass to stellar wind mass, as a consequence of the evaporation rates dependence on temperature and the lowering of temperature by mass loading. In particular it was difficult to find ratios approaching what was observationally required. The work in this paper complements PDH by considering the case in which hydrodynamic ablation is the dominant mass addition process. As conductively driven evaporation has a very temperature sensitive rate, ablation is likely to regulate clump dispersal into lower temperature media.

Our solutions are again potentially relevant to such diverse objects as WBBs created by a faster wind interacting with a clumpy AGB superwind, by the wind of a young star interacting with surrounding molecular material, and the wind of an active galactic nucleus impacting its environment.

\section{Similarity solutions}

The basic physics behind the hydrodynamic ablation of material from dense clumps into the surrounding flow was presented by Hartquist et al. (1986). It was proposed that if the flow around a clump is subsonic, hydrodynamic mixing occurs as a result of the well-known Bernoulli effect. For flow with a Mach number $M$ with respect to the clump of less than unity, this leads to a volume mass injection rate, $\dot{\rho}$, proportional to $M^{4 / 3}$. For supersonic flow, mixing occurs largely as a result of a low pressure region over the reverse face of the clump, "shadowed" from the flow. In this case the mass injection rate is Mach number independent.

In this paper we present similarity solutions for WBBs mass-loaded by hydrodynamic ablation. Our solution method is very similar to that used by $\mathrm{PDH}$, and we refer the reader to that paper for a discussion of many of the assumptions involved. As a starting point, we also assume the same qualitative shock structure in the WBB as shown in Fig. 1 of PDH: that is a central wind source surrounded by a region of unshocked wind, separated from an outer region of shocked wind by an inner shock. The swept-up ambient medium is assumed to radiate efficiently and collapse to a negligibly thin shell coincident with the contact discontinuity separating the stellar and ambient gas (cf. Dyson \& de Vries 1972).

A central and fundamental difference between the conductive case considered in PDH and the ablative case examined here, however, is that here mass loading may also occur in the unshocked stellar wind. The effect is to heat this part of the flow, which leads to reduced Mach numbers, and weaker jump conditions across the inner shock. We shall see that for high mass loading rates, it is possible for the unshocked mass-loaded wind to connect directly with the contact discontinuity, without the presence of an inner shock.

In this work we assume that the flow can be treated as a single fluid. This requires that the ablated clump material merges with the global flow in the sense that its temperature, velocity, and density approach those of the surrounding tenuous material. Ablation by itself might require that the clump material mix microscopically with the wind to reach the density and temperature of the wind. However, though the mass-loss rate may be controlled by ablation, thermal conductivity will almost certainly be responsible for material, once it is stripped from a clump, reaching the physical state of the tenuous fast flowing medium. Thermal conduction accomplishes this 

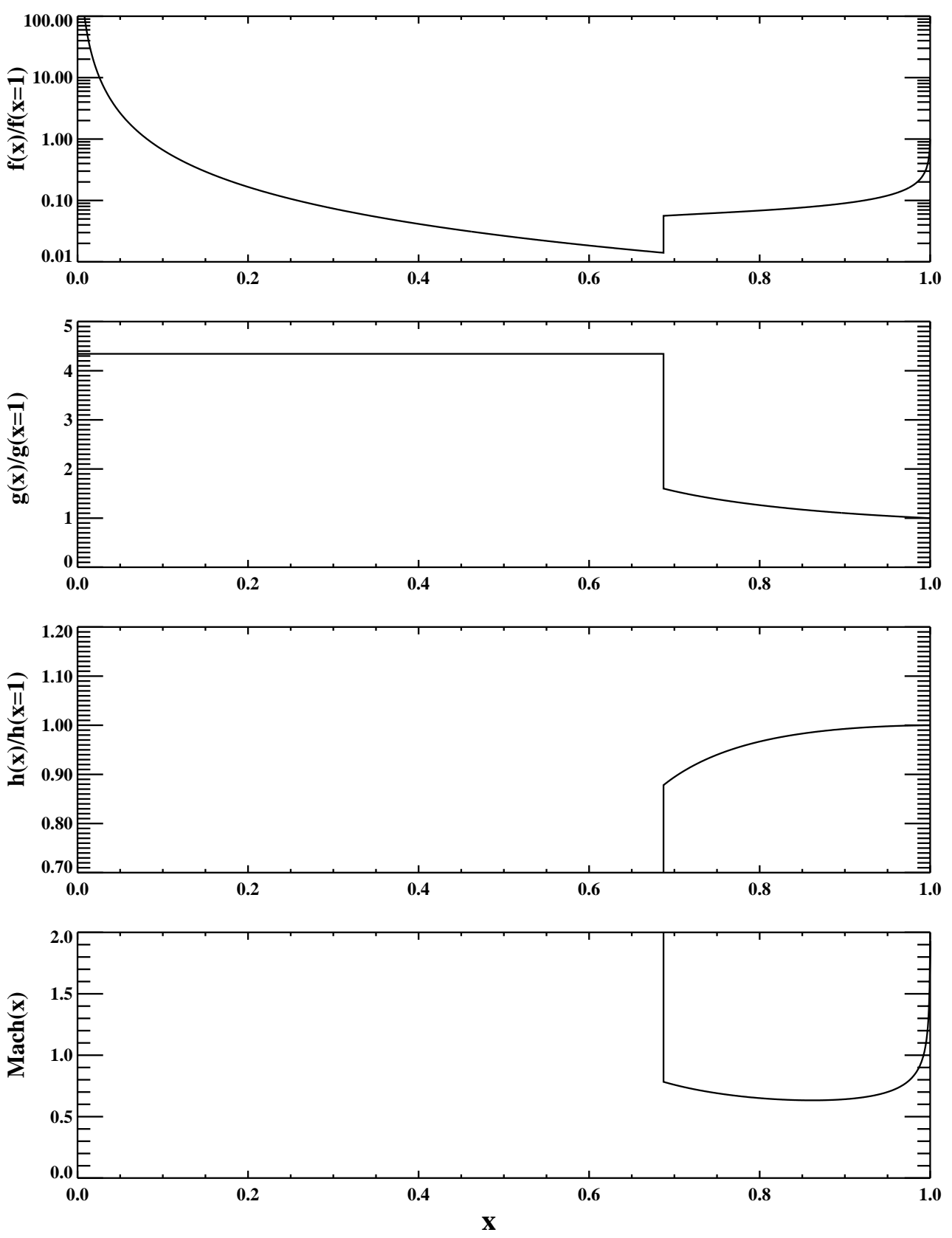

Fig. 1. Results for $\lambda=-3, x_{\text {is }} / x_{\mathrm{cd}}=0.687$, and $\phi=50000$. In the limit of large $\phi$ (i.e. negligible mass loading), the earlier results of Dyson (1973) are recovered. From the top the panels indicate density, velocity, internal energy, and Mach number (with respect to the stationary clumps) as a function of radius. Except for the Mach number, the panels are normalized to values of 1.0 at the contact discontinuity.

phase transition without microscopic mixing, and acceleration to the global flow speed is effected by the response of stripped material to pressure gradients and viscous coupling, which may arise from a host of mechanisms including turbulence. Thus we envisage a two-stage process in which ablation controls the rate at which mass is stripped from the clump but conductivity becomes important for the merging into the global flow.

This receives support from the comparison of the conductively driven evaporation time of a spherical clump with $n T=10^{7} \mathrm{~cm}^{-3}$ and $T=10^{4} \mathrm{~K}$, embedded in a medium with the same pressure (which is typical of planetary nebulae, Wolf-Rayet wind-blown bubbles, and starburst superwinds) and $T=10^{7} \mathrm{~K}$ (which is also typical of hot material in such regions) to the sound crossing time in the clump. The sound crossing time is somewhat (but not hugely) smaller than the conductively driven evaporation time for a large range of clump sizes, if the Cowie \& McKee (1977) estimate of the mass-loss rate driven by saturated conduction is used. Consequently, ablation initiates mass-loss because it causes an increase in the surface area of a clump triggering more conductive heat transfer. Use 
of the analysis of Cowie \& McKee (1977) and McKee \& Cowie (1977) shows that for the assumptions given above, radiative losses do not hinder conductively driven evaporation unless the clump radius is greater than $\approx 15 / n_{\mathrm{f}} \mathrm{pc}$ where $n_{\mathrm{f}}$ is the number density $\left(\mathrm{cm}^{-3}\right)$ of the global tenuous flow. Of course, a clump that does not cool radiatively after it is compressed by a shock will have a shorter sound crossing time and be ablated more rapidly. However, by assuming a clump temperature of $10^{4} \mathrm{~K}$ above we have established that the two-stage process is likely to be relevant in many environments if a clump radiatively cools after being shocked.

Observations of WBBs and PNe indicate that mass loading may not necessarily begin at zero distance from the wind source. For instance, in the young nebula Abell 30 the clumps appear almost all of the way down to the central star (Borkowski et al.1995), whilst in the more evolved Helix Nebula the clumps are all located closer to its edge (e.g. Meaburn et al.1996). We therefore include the radius at which mass-loading "switches on" as a free parameter in our models, and assume that the wind undergoes free expansion interior to this radius. Hence each solution will consist of a region of supersonic wind with no mass loading and an adjacent region with mass loading.

One can imagine two possible causes for this minimum "mass-loading" radius. In one scenario the clumps could have been ejected at low velocity from the central star at an earlier evolutionary stage. The ejection of clumps then abruptly stopped, so that at the time of observation they had travelled a finite distance from the central star. By this process a central region clear of clumps surrounded by a clumpy region can be generated. A second possibility is that clumps interior to the mass-loading radius have been completely destroyed by the wind. It seems reasonable to suppose that clumps located closest to the central star will be destroyed first, since they will have been subjected to the wind from the central star for the longest time. Then as the bubble or nebula evolves, clumps at ever increasing distance from the central star will be destroyed. Timescales for the destruction of clumps by ablation can be estimated from Hartquist et al. (1986) and Klein et al. (1994). Estimated destruction timescales vary from significantly less than to greater than the age of the bubble/PNe, in accord with the different spatial distribution of clumps in objects of differing age.

Regardless of which of the above scenarios is responsible for the existence of such a minimum mass-loading radius, this radius will physically increase with time. Our similarity solution requires that it increases in the same way as that of the contact discontinuity (i.e. $r \propto t^{2 /(5+\lambda)}$, where $\lambda$ is the radial dependence of the mass-loading). For most of the solutions presented in this paper, the minimum mass-loading radius scales with or close to $t$. Since on physical grounds we might expect it to scale as $t$, our solutions closely match this requirement.

In our solutions an inner shock may or may not be present - in the latter case the mass-loaded wind directly connects to the contact discontinuity, and the mass loading may be strong enough for the wind to become subsonic with respect to the clumps before the contact discontinuity is reached. If an inner shock is present, the postshock flow is by definition subsonic with respect to the shock, but may still be supersonic with respect to the clumps. In this case a number of different profiles for the Mach number are possible before the contact discontinuity is reached.

At the center of the bubble prior to the onset of mass loading, we solve only the continuity and momentum equations, with the implicit assumption that the thermal energy of the flow is negligible, whilst in the mass loading regions we additionally solve the energy equation, and include a source term for mass injection in the continuity equation. For a $\gamma=5 / 3$ gas, the equations for the massloaded flow are:

$$
\begin{aligned}
& \frac{\partial \rho}{\partial t}+\nabla \cdot(\rho u)=\dot{\rho} \\
& \frac{\partial(\rho u)}{\partial t}+\nabla \cdot\left(\rho u^{2}\right)+\frac{\partial P}{\partial r}=0 \\
& \frac{\partial\left(\frac{1}{2} \rho u^{2}+\varepsilon\right)}{\partial t}+\nabla \cdot\left(\rho u\left(\frac{1}{2} u^{2}+\frac{5}{3} \frac{\varepsilon}{\rho}\right)\right)=0 .
\end{aligned}
$$

In these equations the symbols have their usual meanings. In the next sections we discuss appropriate similarity variables for these equations, our treatment of the boundary conditions, and the scaling relationships to normalize the resulting solutions. The reader is again referred to $\mathrm{PDH}$ for a more in-depth discussion of the details.

\subsection{The similarity variables}

Let the interclump ambient medium have a density of the form $\rho=\rho_{0} r^{\beta}$, and let us consider the case in which the mass-ablation rate is also radially dependent: $\dot{\rho}=Q r^{\lambda} M^{4 / 3}$ for subsonic ablation $(M<1)$, and $\dot{\rho}=Q r^{\lambda}$ for supersonic ablation $(M>1)$ (cf. Hartquist et al. 1986). A similarity solution demands that $\lambda=(2 \beta-5) / 3$, and the "physical" parameters $r, \rho, u$ and $\varepsilon$ may be expressed in terms of the dimensionless similarity variables $x, f(x)$, $g(x)$, and $h(x)$ where

$$
\begin{aligned}
& r=x Q^{-1 /(5+\lambda)} \dot{E}^{1 /(5+\lambda)} t^{2 /(5+\lambda)} \\
& \rho=Q^{5 /(5+\lambda)} \dot{E}^{\lambda /(5+\lambda)} t^{(5+3 \lambda) /(5+\lambda)} f(x) \\
& u=Q^{-1 /(5+\lambda)} \dot{E}^{1 /(5+\lambda)} t^{-(3+\lambda) /(5+\lambda)} g(x) \\
& \varepsilon=Q^{3 /(5+\lambda)} \dot{E}^{(2+\lambda) /(5+\lambda)} t^{-(1-\lambda) /(5+\lambda)} h(x) .
\end{aligned}
$$

Upon substituting the above similarity variables, the hydrodynamic equations for the region of freely expanding wind become:

$$
\begin{gathered}
\left(g-\frac{2 x}{5+\lambda}\right) f^{\prime}+f g^{\prime}+\frac{2 f g}{x}+\frac{5+3 \lambda}{5+\lambda} f=0 \\
\left(g-\frac{2 x}{5+\lambda}\right) f^{\prime}+\left(2 f-\frac{2 x f}{(5+\lambda) g}\right) g^{\prime}+\frac{2 f g}{x}+\frac{2+2 \lambda}{5+\lambda} f=0
\end{gathered}
$$


where a prime denotes derivation with respect to $x$. For the flow in the mass-loaded region we obtain:

$$
\begin{aligned}
\left(g-\frac{2 x}{5+\lambda}\right) f^{\prime}+f g^{\prime} & +\frac{2 f g}{x}+\frac{5+3 \lambda}{5+\lambda} f \\
& -x^{\lambda} M^{4 / 3}=0 \\
\left(g-\frac{2 x}{5+\lambda}\right) g^{\prime}+\frac{2}{3 f} h^{\prime} & -\frac{3+\lambda}{5+\lambda} g \\
+ & \frac{g}{f} x^{\lambda} M^{4 / 3}=0 \\
\left(g-\frac{2 x}{5+\lambda}\right) h^{\prime}+\frac{5}{3} h g^{\prime} & -\frac{1-\lambda}{5+\lambda} h \\
+ & \frac{10}{3} \frac{h g}{x}-\frac{g^{2}}{2} x^{\lambda} M^{4 / 3}=0
\end{aligned}
$$

where the Mach number, $M=g \sqrt{9 f /(10 h)}$. It is simple to rearrange these equations to find $f^{\prime}, g^{\prime}$, and $h^{\prime}$ which may then be integrated to obtain solutions.

\subsubsection{Boundary conditions}

The values of $f$ and $g$ at $x=\Delta x$, the inner boundary of our integration, are $g=\sqrt{2 / \phi}$ and $f=1 /\left(2 \pi \Delta x^{2} g^{3}\right)$, where $\phi$ is a parameter which specifies the relative amount of mass loading in the solution. $h$ is set to zero at this point. Equations (8) and (9) are integrated to the radius at which mass loading begins. From this point onwards we then integrate Eqs. (10)-(12) instead. The Mach number of the wind rapidly decreases from its initial value of infinity and both the density and velocity of the wind respond to the mass addition. Integration proceeds until the contact discontinuity (CD) is reached. This occurs when the flow velocity is equal to the coordinate velocity (i.e. $v=\mathrm{d} R / \mathrm{d} t)$, which is where $g\left(x=x_{\mathrm{cd}}\right)=2 x /(5+\lambda)$. As previously discussed, this can sometimes occur before the specified position of the inner shock $\left(x=x_{\text {is }}\right)$ is reached, in which case the mass-loaded flow connects directly to the CD. If an inner shock is present, its velocity with respect to the bubble center is $g_{\mathrm{s}}=2 x_{\text {is }} /(5+\lambda)$, and the Mach number of the preshock flow with respect to the shock is

$M_{1}=\left(g_{1}-g_{\mathrm{s}}\right) \sqrt{\frac{f_{1}}{\gamma(\gamma-1) h_{1}}}$

where the subscript "1" indicates the preshock values. Because (unlike for the evaporative case) $M_{1}$ is not necessarily large, we cannot assume that the strong shock jump conditions apply and therefore must use the full RankineHugoniot relations. The postshock values are then:

$$
\begin{aligned}
& f_{2}=\frac{\gamma+1}{\gamma-1} \frac{M^{2}}{M^{2}+2} f_{1} \\
& g_{2}=x_{\mathrm{is}}+\left(g_{1}-x_{\mathrm{is}}\right) \frac{f_{1}}{f_{2}} \\
& h_{2}=\frac{2 \gamma M^{2}-(\gamma-1)}{\gamma+1} h_{1} .
\end{aligned}
$$

Even if the flow velocity has not converged to the coordinate velocity, an inner shock may not exist. This can occur if the mass loading interior to the proposed position of the inner shock is strong, resulting in $M_{1}$ being less than unity. In such a scenario, the jump conditions are not applied and the integration proceeds as before.

At the CD, we must also satisfy conservation of momentum. We take

$\theta \equiv \frac{Q^{-1 /(5+\lambda)} \dot{E}^{1 /(5+\lambda)}}{\rho_{0}^{-1 /(5+\beta)} \dot{E}^{1 /(5+\beta)}}$

as a measure of the ablated mass to the mass in the sweptup shell. It follows that at the contact discontinuity,

$\theta=\left(\frac{(\gamma-1) h}{x^{\beta}\left[g^{2}-\frac{3+\lambda}{(5+\lambda)(3+\beta)} x g\right]}\right)^{1 /(5+\beta)}$

The rate of massloss from the star is

$\dot{M}_{\mathrm{c}}=\lim _{x \rightarrow 0} 4 \pi x^{2} f g \Xi$

where

$\Xi=Q^{2 /(5+\lambda)} \dot{E}^{(3+\lambda) /(5+\lambda)} t^{(6+2 \lambda) /(5+\lambda),}$

which differs from the definition in the conductively driven case. The mass loading parameter $\phi$, which is a measure of the ratio of ablated mass to wind mass is given by $\phi=$ $\dot{M}_{\mathrm{c}} / \Xi$.

\subsection{Scale transformation and normalization}

The dimensionless Eqs. (10)-(12) are invariant under the following transformation, which we shall call a normalization:

$$
\left.\begin{array}{l}
x \rightarrow \alpha x \\
f \rightarrow \alpha^{\lambda} f \\
g \rightarrow \alpha g \\
h \rightarrow \alpha^{2+\lambda} h
\end{array}\right\}
$$

These can be combined to obtain the normalizations for the mass, and for the kinetic and internal energies of the bubble:

$$
\begin{array}{ll}
m=4 \pi \int f x^{2} \mathrm{~d} x & {\left[\alpha^{3+\lambda}\right]} \\
k=4 \pi \int \frac{1}{2} f g^{2} x^{2} \mathrm{~d} x & {\left[\alpha^{5+\lambda}\right]} \\
i=4 \pi \int h x^{2} \mathrm{~d} x & {\left[\alpha^{5+\lambda}\right] .}
\end{array}
$$

The full equation for the mass in the bubble is:

$$
M_{\mathrm{b}}=4 \pi \alpha^{3+\lambda} \Xi t \int_{x=0}^{x_{\mathrm{cd}}} f x^{2} \mathrm{~d} x=\alpha^{3+\lambda} \frac{\dot{M}_{\mathrm{c}} t}{\phi} m .
$$

The total mass lost by the star during its lifetime is

$M_{\mathrm{w}}=\int \dot{M}(t) t=\frac{5+\lambda}{11+3 \lambda} \dot{M}_{\mathrm{c}} t$. 
Table 1. The influence of $x_{\mathrm{ml}}$ on solutions with $\lambda=-3$. Two cases are considered: minor mass loading $(\phi=100)$ whereby the value of $x_{\mathrm{ml}}$ is relatively unimportant, and appreciable mass loading $(\phi=10)$ where $x_{\mathrm{ml}}$ exerts a major influence. From left to right, the columns indicate: i) the value of $x_{\mathrm{ml}}$, ii) the ratio of the shock radii and $\mathrm{CD}$, iii-vi) the fractions of energy that are thermal and kinetic in the bubble, kinetic in the swept-up shell, and radiated, vii) the value of $\theta$, viii) the ratio of mass within the bubble to the total mass injected by the wind (values greater than unity indicate mass loading), ix) the ratio of mass in the swept-up shell to mass in the bubble, $\mathrm{x}$ ) the preshock Mach number of the unshocked wind.

\begin{tabular}{lccccccccc}
\hline$x_{\mathrm{ml}}$ & $x_{\mathrm{is}} / x_{\mathrm{cd}}$ & $I E_{\mathrm{b}}$ & $K E_{\mathrm{b}}$ & $K E_{\mathrm{sh}}$ & $E_{\mathrm{rad}}$ & $\theta$ & $\Phi_{\mathrm{b}}$ & $M_{\mathrm{sh}} / M_{\mathrm{b}}$ & $M_{1}$ \\
\hline$\lambda=-3, \phi=100$ & & & & & & & & & \\
0.1 & 0.271 & 0.46 & 0.014 & 0.469 & 0.059 & 102 & 1.454 & 376 & 4.79 \\
0.2 & 0.268 & 0.46 & 0.013 & 0.469 & 0.059 & 101 & 1.371 & 390 & 4.79 \\
0.33 & 0.266 & 0.46 & 0.013 & 0.469 & 0.059 & 99.7 & 1.289 & 408 & 5.00 \\
0.5 & 0.268 & 0.46 & 0.013 & 0.469 & 0.059 & 99.2 & 1.223 & 426 & 5.56 \\
0.9 & 0.264 & 0.46 & 0.013 & 0.469 & 0.059 & 99.0 & 1.152 & 450 & 11.9 \\
\hline$\lambda=-3, \phi=10$ & & & & & & & & & \\
0.02 & 0.82 & 0.17 & 0.63 & 0.18 & 0.022 & 2.95 & 6.50 & 0.51 & 1.60 \\
0.1 & 0.76 & 0.22 & 0.53 & 0.22 & 0.028 & 2.95 & 4.48 & 0.80 & 1.45 \\
0.2 & 0.73 & 0.24 & 0.49 & 0.24 & 0.030 & 2.90 & 3.84 & 0.92 & 1.34 \\
0.5 & 0.66 & 0.25 & 0.45 & 0.27 & 0.033 & 2.67 & 2.55 & 1.23 & 1.12 \\
0.9 & 0.67 & 0.24 & 0.39 & 0.33 & 0.041 & 2.85 & 1.75 & 2.17 & 2.53 \\
\hline
\end{tabular}

The degree of mass loading in the bubble, $\Phi_{\mathrm{b}}$, is defined as the ratio $M_{\mathrm{b}} / M_{\mathrm{w}}$. The mass-swept up into the shell, $M_{\mathrm{sh}}$, the ratio of swept-up mass to bubble mass, $M_{\mathrm{sh}} / M_{\mathrm{b}}$, the kinetic energy of the shell, $K E_{\mathrm{sh}}$, and the $p d V$ work on the shocked ambient gas, are all identical to the corresponding forms in $\mathrm{PDH}$.

\subsection{Solution procedure}

If an inner shock exists, three main parameters determine the form of a given similarity solution $\left(\lambda, \phi\right.$ and $\left.x_{\mathrm{is}} / x_{\mathrm{cd}}\right)$. However, the ratio of the radius of the onset of mass loading to the radius of the inner shock is an additional parameter unique to the ablative solutions, and its value $\left(x_{\mathrm{ml}}\right)$ may also influence the form of the solution. We find that if the mass loading of the bubble is minor, $x_{\mathrm{ml}}$ has relatively little influence, and vice-versa (since $\dot{\rho} \propto r^{\lambda}$ and $-3 \lesssim \lambda \lesssim-2$ for most of the solutions presented, most of the mass-loading occurs close to the minimum massloading radius). Alternatively, if an inner shock does not exist, $x_{\mathrm{is}} / x_{\mathrm{cd}}$ and $x_{\mathrm{ml}}$ have no meaning as we have defined them. In this case we report the ratio of the onset radius of mass loading to the radius of the contact discontinuity as the parameter $x_{\mathrm{ml}}$.

The ratio of mass pick-up from the clumps to the mass swept-up from the interclump medium (which is related to the value of $\theta$ ) is obtained only after a particular solution has been found. For bubbles whose evolution is significantly altered by mass loading, we require that simultaneously $\phi$ and $\theta$ are both small.

The similarity equations were integrated with a fifthorder accurate adaptive step-size Bulirsch-Stoer method using polynomial extrapolation to infinitesimal step size. Once the $\mathrm{CD}$ was reached, the similarity variables were rescaled using the relationships defined in Eq. (21) so that $x_{\mathrm{cd}}=1$. The mass, and kinetic and thermal energies of the bubble were calculated, as were the kinetic energy of the shell and the energy radiated from it. The correct normalization to satisfy global energy conservation was then obtained. Finally, for given values of $\dot{E}, Q$ and $t$, the similarity variables $x, f, g$ and $h$ may be scaled into the physical variables $r, \rho, u$ and $\varepsilon$.

\section{Results}

We first checked our work against the solutions obtained by Dyson (1973) for WBBs with no mass loading. For this comparison it was required that $\lambda=-3$ and that $\phi$ was large. For $\lambda=-3$ the radius of minimum mass loading increases as $t$. Excellent agreement with Dyson (1973) was found over a large range of $x_{\mathrm{is}} / x_{\mathrm{cd}}$. For large $\phi$ (i.e. negligible mass loading) the value of $x_{\mathrm{ml}}$ has no effect on the resulting solution. Figure 1 shows a sample solution.

In Fig. 2 we show results for $\lambda=-3, \phi=100$, with different values of $x_{\mathrm{ml}}$. The mass loading in these results is small, but not negligible, so that the precise value of $x_{\mathrm{ml}}$ has minor consequences for the solution obtained. In Table 1 we tabulate important parameters from these solutions. In particular, we find that values for the ratio $x_{\text {is }} / x_{\text {cd }}$ and the energy fractions are very similar. The fractional mass loading of the bubble, $\Phi_{\mathrm{b}}$, is somewhat more sensitive to the value of $x_{\mathrm{ml}}$, and increases when the "turnon" radius for mass loading is decreased, as one would expect. The preshock Mach number also reflects the degree of preshock mass loading, again as expected. Interestingly, the profiles of postshock Mach number are, however, very insensitive to the preshock Mach number. With the assumption of constant $\phi$, we note that since $x_{\text {is }} / x_{\mathrm{cd}}$ varies for the above results it was important to see whether this was a possible factor for some of the differences in these solutions. Therefore we also investigated the effect of different values of $x_{\mathrm{ml}}$ whilst keeping $x_{\mathrm{is}} / x_{\mathrm{cd}}$ constant and varying $\phi$ as necessary. We find that the resulting 

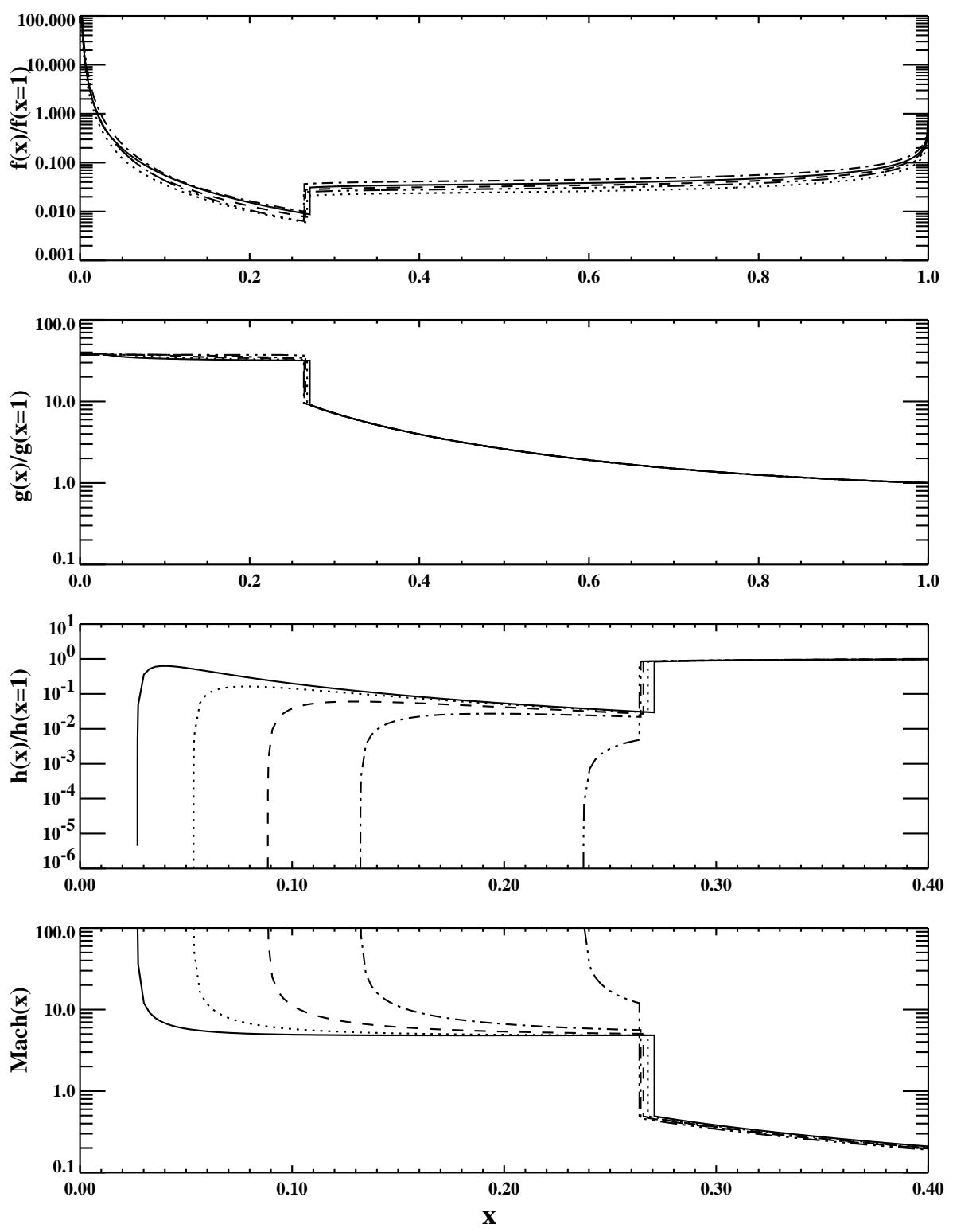

Fig. 2. Results for $\lambda=-3, \phi=100$, and various values of $x_{\mathrm{ml}}$. For these parameters the mass loading is small but non-negligible, and hence demonstrates $x_{\mathrm{ml}}$ having a minor influence on the results. Results for five values of $x_{\mathrm{ml}}$ are shown: 0.1 (solid), 0.2 (dots), 0.33 (dashes), 0.5 (dot-dash), and 0.9 (dot-dot-dot-dash). The panels are the same as in Fig. 1. In Fig. 3 we show solutions where the value of $x_{\mathrm{ml}}$ has a much greater influence.

solutions are again fairly similar, and differ at about the same level as the results in the top half of Table 1. Therefore it seems that varying $x_{\mathrm{ml}}$ has a direct effect on the results, and not just an indirect influence through changes induced in $x_{\mathrm{is}} / x_{\mathrm{cd}}$ and/or $\phi$. In the following, therefore, we will vary $x_{\mathrm{ml}}$ whilst keeping $\phi$ fixed.

At the other extreme, Fig. 3 shows results where the value of $x_{\mathrm{ml}}$ can have large consequences for the solutions obtained. This occurs for values of $\phi$ for which the resulting mass loading of the bubble is important. Parameters from these solutions are tabulated in the lower half of Table 1 . Although the ratio $x_{\text {is }} / x_{\mathrm{cd}}$ remains relatively insensitive to the value of $x_{\mathrm{ml}}$, the fractional mass loading $\Phi_{\mathrm{b}}$, the ratio of the shell mass to the bubble mass
$M_{\mathrm{sh}} / M_{\mathrm{b}}$, and the energy partition are all significantly affected, as Table 1 shows. This is expected given that most of the mass loading occurs near the minimum mass loading radius for $\lambda=-3$.

An interesting consequence of mass loading the wind prior to the inner shock is that if the mass loading is large, the Mach number of the flow can be reduced so much that the flow directly connects to the contact discontinuity without the presence of an inner shock. In Fig. 4 we show one solution where this happens. In this example, the flow is continuously supersonic with respect to the clumps, and we obtain $\Phi_{\mathrm{b}}=1.25$ and $M_{\mathrm{sh}} / M_{\mathrm{b}}=0.07$. This perhaps indicates that this morphology is most favoured at early stages of the evolution of the bubble before 

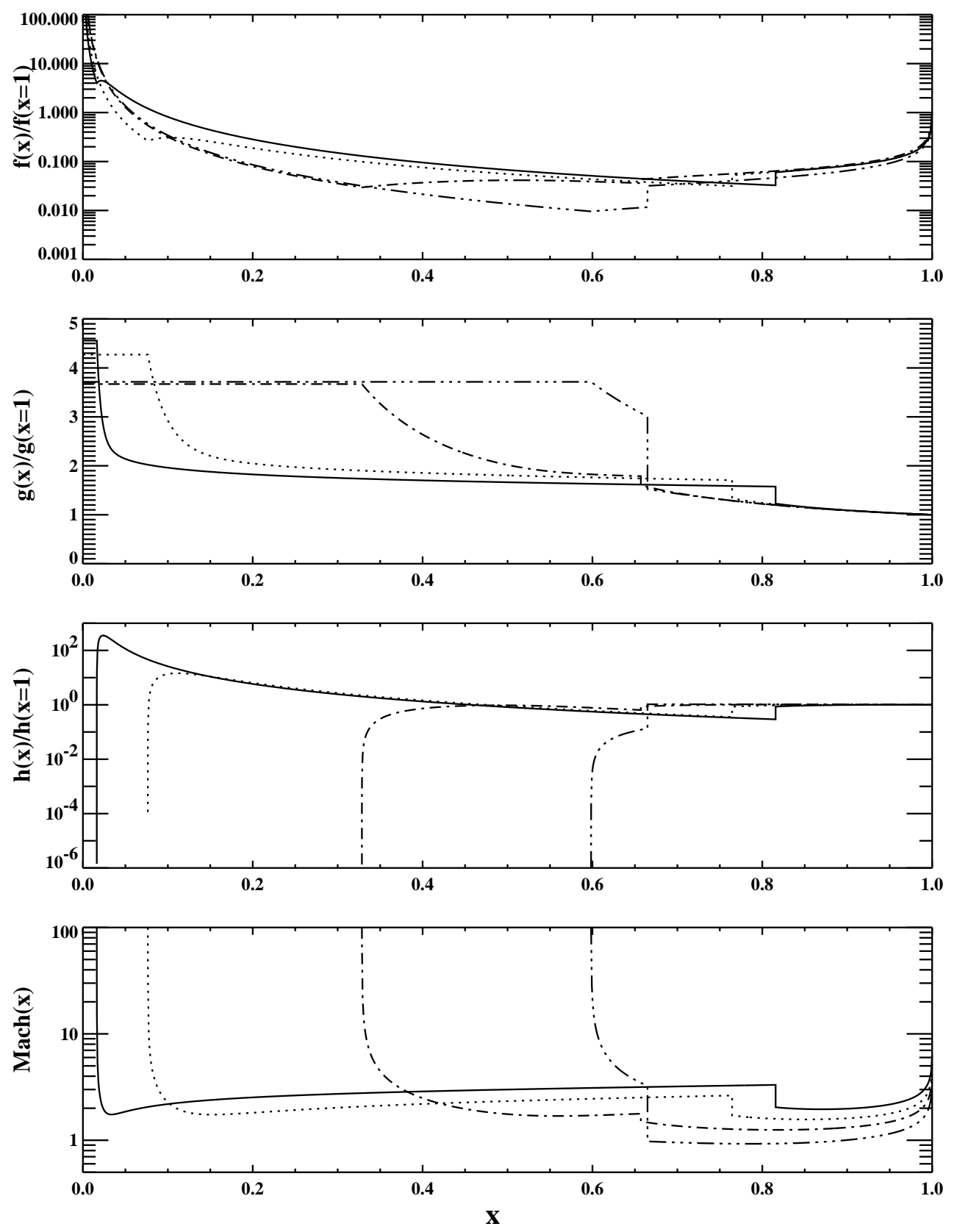

Fig. 3. Results for $\lambda=-3, \phi=10$, and various values of $x_{\mathrm{ml}}$. For this value of $\phi$, the mass loading is appreciable, and hence the solutions shown demonstrate $x_{\mathrm{ml}}$ having a major influence. Results for four values of $x_{\mathrm{ml}}$ are shown: 0.02 (solid), 0.1 (dots), 0.5 (dot-dash), and 0.9 (dot-dot-dot-dash). The panels again represent density, velocity, internal energy and Mach number.

a significant amount of ambient medium is swept-up. In another example of "direct connection", we find a solution where the Mach number of the flow with respect to the clumps drops below unity (see panel $\mathrm{f}$ of Fig. 6). In this case, $\Phi_{\mathrm{b}}=3.4$ and $M_{\mathrm{sh}} / M_{\mathrm{b}}=480$, suggesting a much older bubble. We also find that low values of $\lambda(\approx-3)$ are apparently needed to obtain directly-connected solutions.

In Fig. 5 we compare solution profiles for the case where $\lambda=-2$ and for negligible and high mass loading rates. A $\lambda=-2$ clump distribution is the most physically plausible for many astrophysical objects. For this distribution the minimum mass loading radius increases as $t^{2 / 3}$, which is not far removed from a linear increase with $t$. The solutions have been scaled from the similarity results by assuming current values for the wind parameters appropriate for a WR star: $\dot{M}_{\mathrm{c}}=10^{-5} M_{\odot} \mathrm{yr}^{-1}$ and $v_{\mathrm{w}}=1500 \mathrm{~km} \mathrm{~s}^{-1}$. The mass-loss rate increases with time as $t^{2 / 3}$ whilst the wind velocity decreases as $t^{-1 / 3}$, so the wind has evolved from a case more suitable to an O-star. The assumed bubble age is $t=10^{4} \mathrm{yr}$. The interclump medium varies as $\rho \propto r^{-1 / 2}$ (i.e. $\beta=-1 / 2$ ) which is a flatter distribution than for a constant velocity wind $\left(r^{-2}\right)$. However, since the wind mass-loss rate is increasing and the velocity decreasing, an $r^{-1 / 2}$ interclump density profile is physically realistic for such a scenario. In comparison with the negligibly mass-loaded solution, 

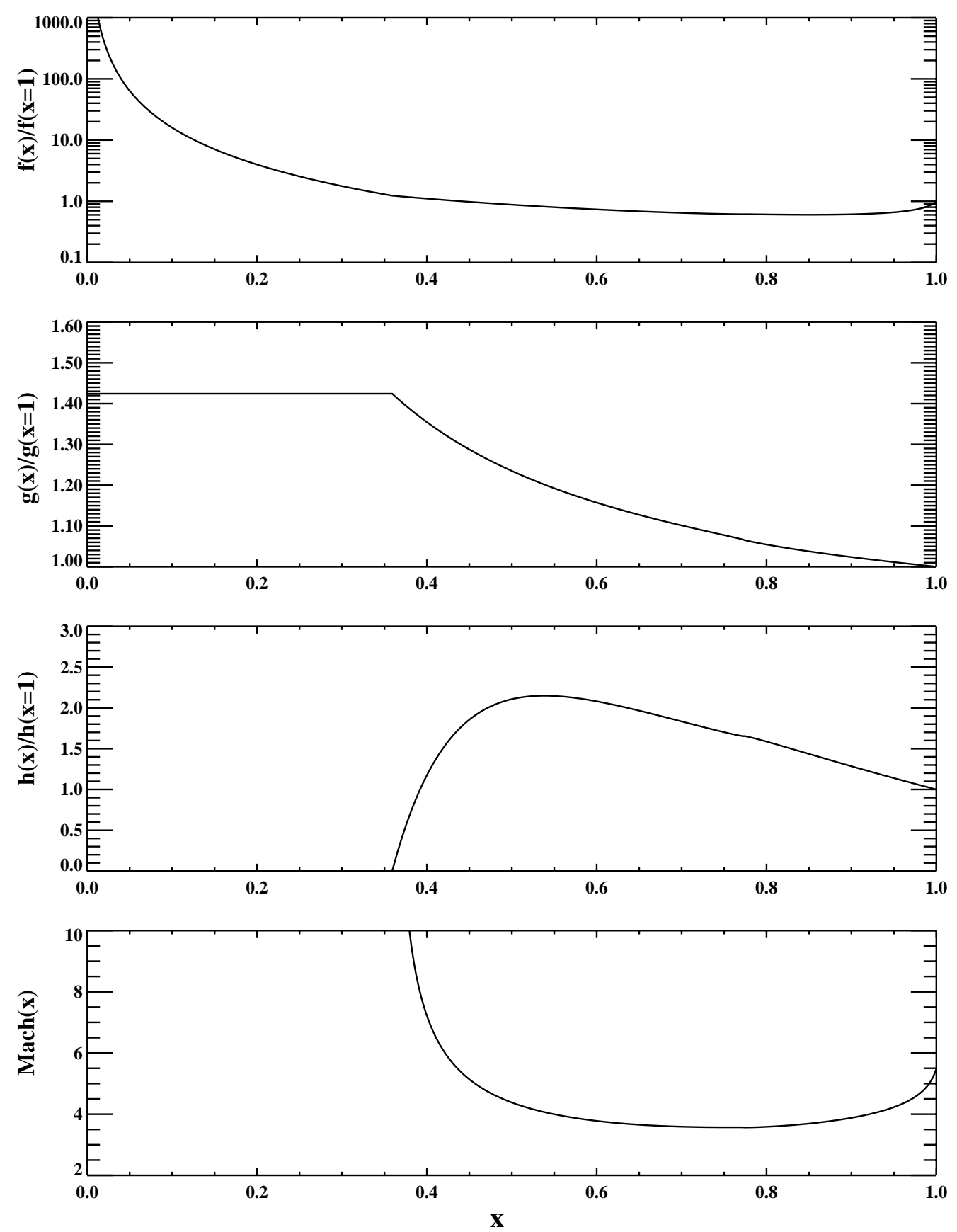

Fig. 4. Results for $\lambda=-3, \phi=50$. Here mass loading is so severe that the flow directly connects to the contact discontinuity. For the solution shown, the onset of mass loading occurs at $x=0.359 x_{\mathrm{cd}}$. Once the flow starts mass loading, its velocity drops, its relative density increases, its kinetic energy drops, and its thermal energy increases.

the increase in density inside the bubble for the highly mass-loaded solution can be clearly seen, along with a decrease in preshock velocity, preshock Mach number, and postshock temperature. Interestingly, the Mach number of the postshock flow is higher for the heavily mass-loaded solution. Note also that the heavily mass-loaded solution extends to a larger bubble radius. This is a consequence of the different preshock interclump densities: for the heavily mass-loaded solution $n_{\mathrm{e}}=0.066$, whilst $n_{\mathrm{e}}=0.195$ for the negligibly mass-loaded solution.
In PDH it was shown that there is a negative feed-back mechanism caused by the evaporation of mass from embedded clumps, which set a maximum limit to the amount that a bubble could be massloaded. Additionally, for the bubble mass to be greater than the mass of the swept-up shell, a large radial dependence of the mass loading was required $(\lambda \geq 4)$. In this work we instead find that small values of $\lambda$ are required to satisfy this condition. Our simulations show that it is impossible to obtain a similarity solution with $M_{\mathrm{sh}}<M_{\mathrm{b}}$ for $\lambda \geq-1$ (e.g. for $\lambda=1$, 

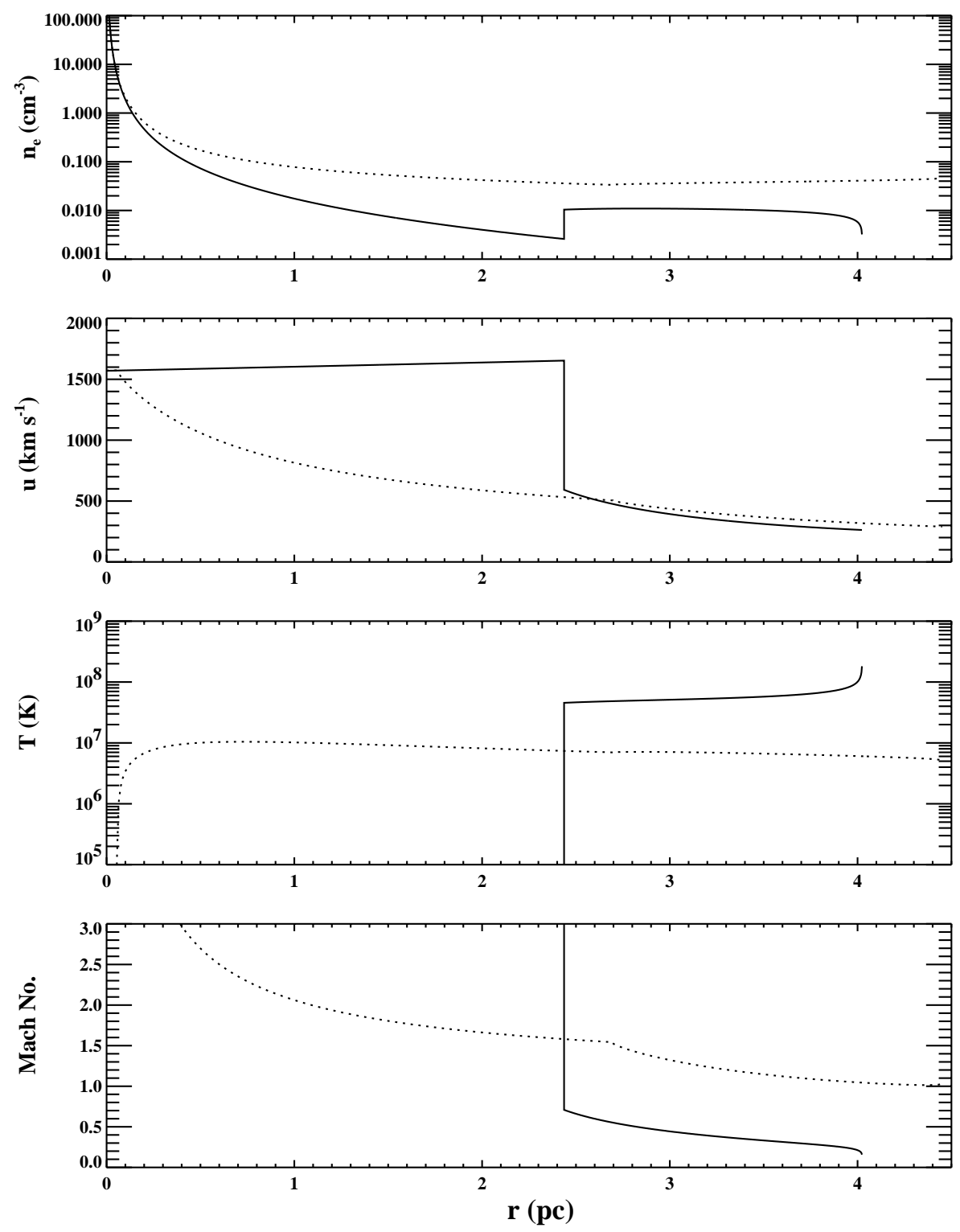

Fig. 5. Comparison of solutions with negligible (solid) and high (dots) mass loading rates, for a given value of $\lambda(-2)$ and $x_{\text {is }} / x_{\mathrm{cd}}$ (0.606). The ratio of swept-up mass to bubble mass is 12.3 and 0.96 respectively, whilst the ratio of ablated mass to wind mass is 7.54 for the mass-loaded solution. The preshock Mach number for the mass-loaded solution is 1.006. In both cases, the similarity solution has been scaled such that the current wind parameters are appropriate for a WR star: $\dot{M}_{\mathrm{c}}=10^{-5} M_{\odot} \mathrm{yr}^{-1}$ and $v_{\mathrm{w}}=1500 \mathrm{kms}^{-1}$.

$\left.M_{\mathrm{sh}} \gtrsim 2.4 M_{\mathrm{b}}\right)$. However, for smaller values of $\lambda$, solutions satisfying $M_{\mathrm{sh}}<M_{\mathrm{b}}$ can be found (e.g. for $\lambda=-2(-3)$, we can obtain $M_{\mathrm{b}} \approx 8(29) M_{\mathrm{sh}}$, where $\left.\Phi_{\mathrm{b}} \approx 7.6(15)\right)$. For a given value of $M_{\mathrm{sh}} / M_{\mathrm{b}}$ there appears to be a maximum value for $\Phi_{\mathrm{b}}$. It occurs when mass loading starts very close to the bubble centre (i.e. $x_{\mathrm{ml}} \rightarrow 0$ ) and when the flow remains supersonic relative to the clumps over the entire bubble radius.

We have also investigated the Mach number profile of the solutions that we obtain, of which some are already shown in Figs. 2 and 3. As for the evaporative mass loading in $\mathrm{PDH}$, we note that a number of different forms are possible, and summarize these in Fig. 6 .
Finally, radial profiles of the X-ray emissivity per unit volume and temperature have been calculated (Fig. 7). We assume that the emissivity $\Lambda \propto n^{2} T^{-1 / 2}$, which is a good approximation over the temperature range $5 \times 10^{5} \mathrm{~K} \lesssim$ $T \lesssim 5 \times 10^{7} \mathrm{~K}$ (cf. Kahn 1976). The upper panels show results where an inner shock is present, and we will discuss these first. The solution with the solid line displays relatively constant values of $\Lambda$ and $T$, and is characterized by a high degree of mass loading $\left(\Phi_{\mathrm{b}}=9.7\right)$. Note that the highest temperature in the bubble does not occur postshock, but rather shortly after mass loading begins. The very high emissivity at the onset of mass loading is a consequence of the low temperature at this point and the assumed $T^{-1 / 2}$ dependence of our emissivity, and is 

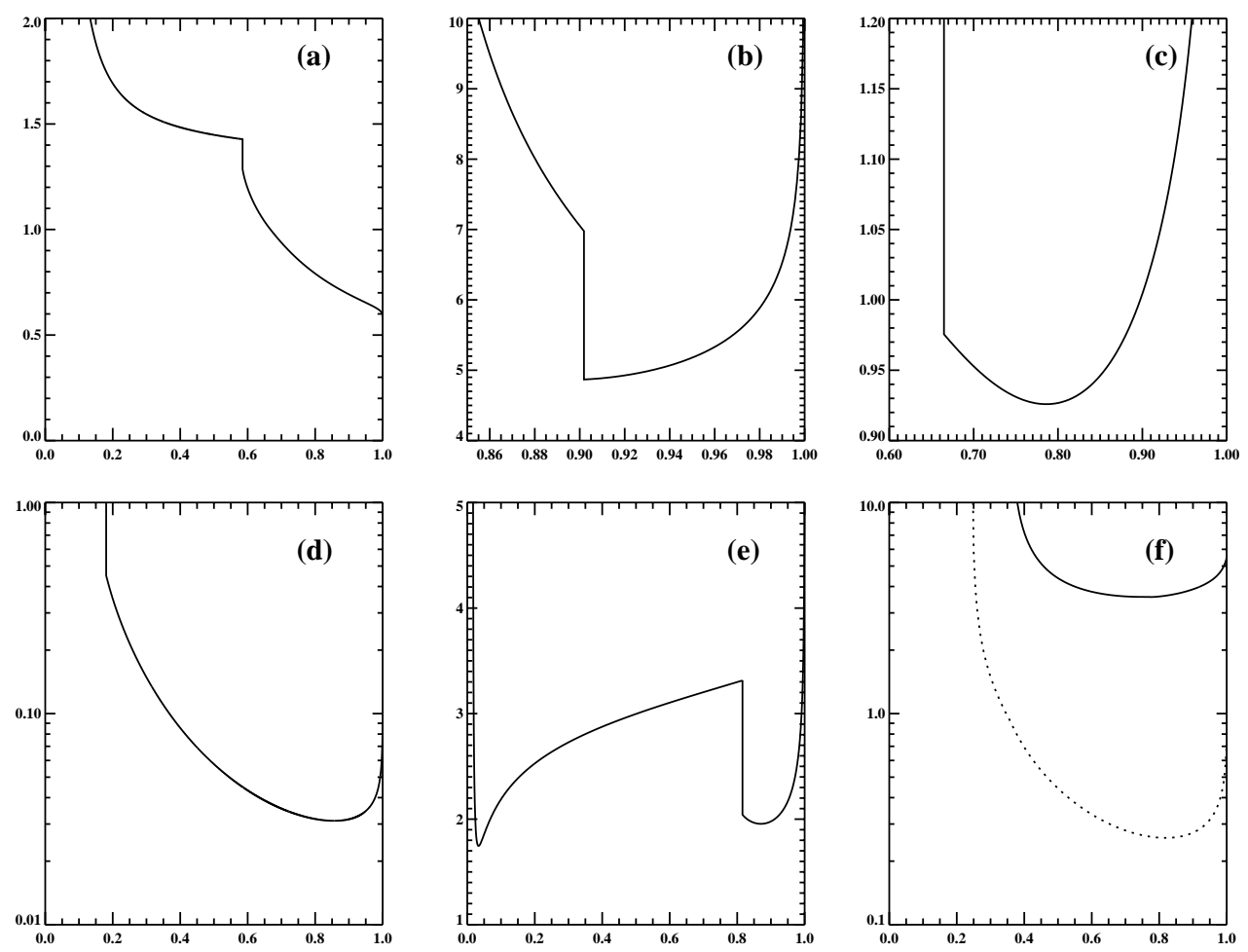

Fig. 6. The Mach number as a function of $x$ for a number of different solutions. With respect to the clumps, the shocked region can be either entirely subsonic, entirely supersonic, or have one (or maybe more) sonic points. The panels show: a) shocked region with a monotonic decrease in Mach number $\left(\lambda=-2, \phi=0.5, x_{\mathrm{is}} / x_{\mathrm{cd}}=0.585, x_{\mathrm{ml}}=0.1\right)$; b) shocked region entirely supersonic with a monotonic increase in Mach number $\left(\lambda=-3, \phi=100, x_{\text {is }} / x_{\mathrm{cd}}=0.902, x_{\mathrm{ml}}=0.9\right)$; c) postshock Mach number initially subsonic and decreasing, before levelling off and subsequently increasing through a sonic point $(\lambda=4, \phi=10$, $\left.\left.x_{\mathrm{is}} / x_{\mathrm{cd}}=0.297, x_{\mathrm{ml}}=0.1\right) ; \mathbf{d}\right)$ shocked region entirely subsonic with a decreasing Mach number followed by a modest increase close to the contact discontinuity $\left(\lambda=-3, \phi=10^{5}, x_{\mathrm{is}} / x_{\mathrm{cd}}=0.181, x_{\mathrm{ml}}=0.1\right)$; e) postshock flow continuously supersonic though with an initial decrease in Mach number $\left(\lambda=-3, \phi=10, x_{\mathrm{is}} / x_{\mathrm{cd}}=0.816, x_{\mathrm{ml}}=0.02\right)$; f $)$ continuously supersonic flow (solid) directly connected to the contact discontinuity (i.e. no inner shock) $\left(\lambda=-3, \phi=50, x_{\mathrm{ml}}=0.359\right)$ and a flow becoming subsonic (dots) directly connected to the contact discontinuity $\left(\lambda=-3.2, \phi=12.5, x_{\mathrm{ml}}=0.246\right)$.

not physical. The solution with the dotted line has an interior dominated by free-expansion of the wind source. Mass loading only occurs over the final 20 per cent of the radius, and is relatively weak, and the maximum temperature in the bubble occurs immediately postshock. The solution with the dashed line has some characteristics of each of the other solutions. It is mass-loaded from small radius which again leads to the maximum bubble temperature occurring near the center. However, the mass loading is less severe $\left(\Phi_{\mathrm{b}}=6.5\right)$ and its radial dependence steeper $(\lambda=-3)$ than for the solution with the solid line, so that there is a large fall in density between the switch-on radius for mass loading and $x_{\text {is }}$. This leads to a steep rise in emissivity from the inner shock to the bubble center in a similar fashion to the solution with the dotted line.

The bottom panels of Fig. 7 show results where there is no inner shock and where the mass loading region directly connects to the contact discontinuity. The Mach number profiles for these solutions are displayed in the bottom-right panel of Fig. 6. As can be seen, the temperature profiles are approximately flat over their respective mass loading regions, and are very similar in shape to each other. This contrasts with the behaviour of their emissivity profiles, where it is clear that the solution represented by the solid (dotted) line has $\Lambda$ generally decreasing (increasing) with radius.

A general result from Fig. 7, which was also found in the evaporatively mass-loaded bubbles of $\mathrm{PDH}$, is that higher mass loading tends to decrease the central emissivity and increase the central temperature relative to the limb.

\subsection{Comparison with observations and other theoretical work}

Although our work cannot be directly compared to the hydrodynamic simulations of Arthur et al. (1993, 1996), a number of similarities exist. These include a sharp increase in temperature at the onset of mass loading, and often $\partial \rho / \partial x>0$ over the mass-loaded region. A noticeable achievement of the work of Arthur et al. (1996) was the reproduction of the observed correlation between the velocity and ionization potential of ultraviolet absorption features towards the central star (Smith et al. 1984). Our solutions also often reproduce this correlation (e.g. see Fig. 5 which shows the velocity and temperature 

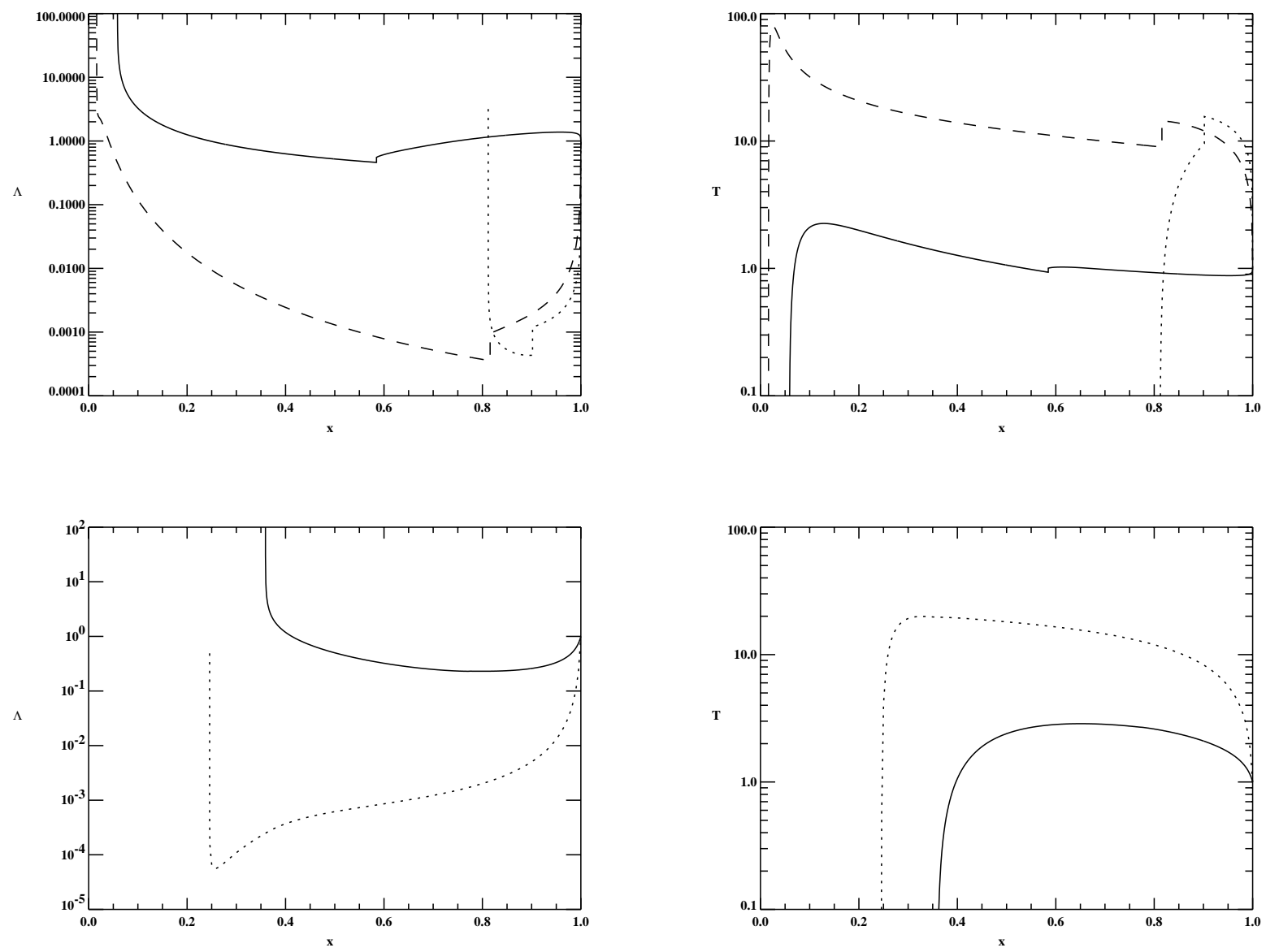

Fig. 7. Radial profiles of emissivity per unit volume and temperature, normalized to values of 1.0 at the limb. The upper panels show results where an inner shock is present, whilst the bottom panels show results where the mass loading region directly connects to the contact discontinuity. For the upper panels the results shown are for: $\lambda=-2, \phi=0.5, x_{\mathrm{is}} / x_{\mathrm{cd}}=0.59, x_{\mathrm{ml}}=0.1$ (solid); $\lambda=-3, \phi=100, x_{\mathrm{is}} / x_{\mathrm{cd}}=0.90, x_{\mathrm{ml}}=0.9(\operatorname{dots}) ; \lambda=-3, \phi=10, x_{\mathrm{is}} / x_{\mathrm{cd}}=0.82, x_{\mathrm{ml}}=0.02$ (dashes). For the lower panels the results are for: $\lambda=-3, \phi=50, x_{\mathrm{ml}}=0.36$ (solid); $\lambda=-3.2, \phi=12.5, x_{\mathrm{ml}}=0.25$ (dots).

decreasing together for the heavily mass-loaded solution), as do those of Hanami \& Sakashita (1987). Interestingly, solutions with mass-loading from conductive evaporation of the swept-up shell do not reproduce this correlation (see Weaver et al. 1977).

\section{Summary}

We have investigated the evolution of a mass-loaded wind blown bubble with a constant rate of injection of mechanical energy from a central wind source. The mass loading occurs due to the hydrodynamic ablation of distributed clumps, and is "switched-on" at a specified radius, interior to which the wind expands freely. The requirement that the solution be self-similar imposes a link between the radial variation of the interclump density $\left(\rho \propto r^{\beta}\right)$ and the rate of mass loading from the clumps $\left(\dot{\rho} \propto r^{\lambda}\right)$ which forces $\lambda=(2 \beta-5) / 3$.

We first produced solutions with negligible mass loading and $\beta=-2$ (which correspond to constant $\dot{M}_{\text {wind }}$ and $v_{\text {wind }}$ ), which we compared with results obtained by
Dyson (1973). Excellent agreement for the structure of the bubble was found. We also confirmed that for negligible mass loading, the value of $x_{\mathrm{ml}}$ had no effect on the resulting solutions, as desired.

We then investigated the changes in the structure of the bubble for different values of $\lambda, \phi, x_{\mathrm{is}} / x_{\mathrm{cd}}$, and $x_{\mathrm{ml}}$. The central conclusions are:

- Substantial mass loading of the wind-blown bubble can occur over a wide range of $\lambda$. However, to additionally satisfy the requirement that the bubble mass is larger than the mass of the swept-up shell, low values of $\lambda$ are needed (e.g. $\lambda \lesssim-2$ ).

- The profiles of the flow variables are significantly altered under conditions of large mass loading. With respect to solutions with negligible mass loading, the density and temperature profiles increase, and the velocity and Mach number profiles drop. Changes to the profiles can be very rapid. Mass loading of the wind also reduces the Mach number prior to the inner shock. 
- The mass-loaded wind may also connect directly to the contact discontinuity without the need for a global inner shock.

- The Mach number of the flow relative to the clumps which are injecting the mass can take several different forms. The flow can be either entirely supersonic, or have one (or maybe more) sonic points.

- Whilst mass loading reduces the bubble temperature, it also tends to reduce the emissivity in the interior of the bubble relative to its limb, whilst simultaneously increasing the central temperature relative to the limb temperature. The maximum temperature in the bubble is often not the post-inner-shock temperature, but occurs near the onset of mass loading. In some cases this can be many times greater than the post-innershock temperature.

Acknowledgements. JMP would like to thank PPARC for the funding of a PDRA position, and Sam Falle and Rob Coker for helpful discussions. We would also like to thank an anonymous referee whose suggestions improved this paper. This work has made use of Nasa's Astrophysics Data System Abstract Service.

\section{References}

Arthur, S. J., Dyson, J. E., \& Hartquist, T. W. 1993, MNRAS, 261,425

Arthur, S. J., Dyson, J. E., \& Hartquist, T. W. 1994, MNRAS, 269, 1117

Arthur, S. J., Henney, W. J., \& Dyson, J. E. 1996, A\&A, 313, 897

Borkowski, K. J., Harrington, J. P., \& Tsvetanov, Z. I. 1995, ApJ, 449, L143
Chièze, J. P., \& Lazareff, B. 1981, A\&A, 95, 194

Cowie, L. L., \& McKee, C. F. 1977, ApJ, 211, 135

David, L. P., \& Durisen, R. H. 1989, ApJ, 346, 618

Durisen, R. H., \& Burns, J. O. 1981, MNRAS, 195, 535

Dyson, J. E. 1973, A\&A, 23, 381

Dyson, J. E., \& de Vries, J. 1972, A\&A, 20, 223

Dyson, J. E., \& Hartquist, T. W. 1987, MNRAS, 228, 453

Dyson, J. E., \& Hartquist, T. W. 1992, Ap. Lett. Comm., 28, 301

Dyson, J. E., Williams, R. J. R., \& Redman, M. P. 1995, MNRAS, 237, 700

Hanami, H., \& Sakahita, S. 1987, A\&A, 181, 343

Hartquist, T. W., Dyson, J. E., Pettini, M., \& Smith, L. J. 1986, MNRAS, 221, 715

Hartquist, T. W., \& Dyson, J. E. 1993, QJRAS, 34, 57

Kahn, F. D. 1976, A\&A, 50, 145

Klein, R. I., McKee, C. F., \& Colella, P. 1994, ApJ, 420, 213

McKee, C. F., \& Cowie, L. L. 1977, ApJ, 215, 213

McKee, C. F., \& Ostriker, J. P. 1977, ApJ, 218, 148

Meaburn, J., Clayton, C. A., Bryce, M., \& Walsh, J. R. 1996, MNRAS, 281, L57

Meaburn, J., Nicholson, R. A., Bryce, M., Dyson, J. E., \& Walsh, J. R. 1991, MNRAS, 252, 535

Pittard, J. M., Dyson, J. E., \& Hartquist, T. W. 2001, A\&A, 367,1000

Smith, L. J., Pettini, M., Dyson, J. E., \& Hartquist, T. W. 1984, MNRAS, 211, 679

Strickland, D. K., \& Stevens, I. R. 2000, MNRAS, 314, 511

Toniazzo, T., Hartquist, T. W., \& Durisen, R. H. 2001, MNRAS, 322, 149

Weaver, R., McCray, R., Castor, J., Shapiro, P., \& Moore, R. 1977, ApJ, 218, 377

Williams, R. J. R., Dyson, J. E., \& Hartquist, T. W. 1999, A\&A, 344, 675

Williams, R. J. R., Hartquist, T. W., \& Dyson, J. E. 1995, ApJ, 446, 759 